\title{
Síndrome metabólica em adolescentes com sobrepeso e obesidade
}

\author{
Metabolic syndrome in obese and overweight adolescents
}

\begin{abstract}
Marilisa Stenghel F. Souza ${ }^{1}$, Renata Barco Leme ${ }^{2}$, Ruth Rocha Franco ${ }^{3}$, Ceres Concilio Romaldini ${ }^{4}$, Rosana Tumas ${ }^{5}$, Ary Lopes Cardoso ${ }^{6}$, Durval Damiani ${ }^{7}$
\end{abstract}

\section{RESUMO}

Objetivo: Estudar a prevalência da síndrome metabólica em adolescentes acompanhados em ambulatório de obesidade.

Métodos: Foram avaliados 84 adolescentes com idades entre dez e 19 anos, divididos em dois grupos, de acordo com o escore Z do índice de massa corpórea ( $Z$ IMC), sendo um o grupo de adolescentes com sobrepeso (GSP) e o outro, o grupo

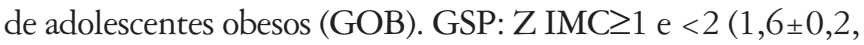

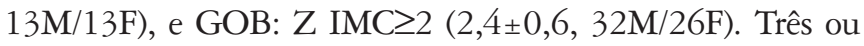
mais dos critérios a seguir foram considerados no diagnóstico da síndrome metabólica e avaliados pelo teste do qui-quadrado entre os grupos: Z IMC $\geq 2$; triglicérides em jejum >130mg/dL; lipoproteína de alta densidade $<35 \mathrm{mg} / \mathrm{dL}$; glicemia em jejum $\geq 100 \mathrm{mg} / \mathrm{dL}$ ou homeostatic model assessment index (HOMA) > 2,5; elevação da pressão arterial acima do percentil 90 ajustada para gênero, estatura e idade.

Resultados: A prevalência da síndrome metabólica esteve significantimente elevada nos adolescentes obesos (GOB: $40 \%$ versus GSP: $4 \%, p=0,0008)$. O grupo GOB mostrou maiores valores referentes a insulinismo ( $54 \%$ versus $19 \%$, $p=0,003)$, HOMA (66\% versus $38 \%, p=0,01)$ e trigliceride$\operatorname{mia}(21 \%$ versus $4 \% ; p=0,04)$.

Conclusões: É importante que o pediatra fique atento aos sinais de síndrome metabólica em adolescentes obesos. A detecção precoce pode ser feita por meio de simples parâmetros e permite a adoção de medidas preventivas para o desenvolvimento da doença cardiovascular em adolescentes.

Palavras-chave: síndrome $\mathrm{X}$; adolescente; obesidade; doenças cardiovasculares; resistência à insulina; hipertensão.

'Mestre em Medicina pela Faculdade de Medicina da Universidade de São Paulo (FMUSP) e médica assistente da Unidade de Nutrologia do Instituto da Criança do Hospital das Clínicas da FMUSP (HCFMUSP) 2Doutora em Nutrição Humana Aplicada pela Faculdade de Ciências Farmacêuticas da USP e mestre em Biodinâmica do Movimento pela Escola de Educação Física e Esportes da USP

${ }^{3}$ Médica pediatra e complementanda da Unidade de Endocrinologia do Instituto da Criança do HCFMUSP

${ }^{4}$ Doutora em Medicina pela FMUSP e médica assistente da Unidade de Nutrologia do Instituto da Criança do HCFMUSP

${ }^{5}$ Mestre em Medicina pela FMUSP e médica assistente da Unidade de Nutrologia do Instituto da Criança do HCFMUSP

${ }^{6}$ Doutor em Medicina pelo Departamento de Pediatria da FMUSP e respon-

\section{ABSTRACT}

Objective: Evaluate the prevalence of metabolic syndrome in adolescents followed in an outpatient obesity clinic.

Methods: 84 adolescents (ten to 19 years old) were divided in two groups, one composed by overweight adolescents $(\mathrm{OWG})$ and the other, by obese adolescents (OBG), according to $\mathrm{Z}$ scores of the body mass index (Z BMI). OWG: $\mathrm{Z} \mathrm{BMI} \geq 1$ and $<2(1.6 \pm 0.2 ; 13 \mathrm{M} / 13 \mathrm{~F})$ and $\mathrm{OBG}: \mathrm{Z} \mathrm{BMI} \geq 2(2.4 \pm 0.6$; $32 \mathrm{M} / 26 \mathrm{~F})$. Three or more criteria were considered to define the presence of metabolic syndrome and tested by chi-square: Z BMI $\geq 2$; fasting triglycerides $>130 \mathrm{mg} / \mathrm{dL}$; high-density lipoprotein cholesterol $<35 \mathrm{mg} / \mathrm{dL}$; fasting glucose $>100 \mathrm{mg} / \mathrm{dL}$ or homeostatic model assessment index (HOMA) $>2.5$ and blood pressure above the $90^{\text {th }}$ percentile, adjusted for age, gender and length.

Results: The prevalence of metabolic syndrome was significantly elevated in obese adolescents (OBG: $40 \%$ versus OWG: $4 \%, p=0.0008$ ). The major differences between groups were observed regarding the presence in OBG adolescents of hyperinsulinism ( $54 \%$ versus $19 \%, p=0.003$ ), HOMA ( $66 \%$ versus $38 \%, p=0.01$ ) and hypertriglyceridemia ( $21 \%$ versus $4 \%, p=0.04)$.

Conclusions: It is important to be aware of the early signs of metabolic syndrome in obese adolescents, which can be screened by simple techniques. As the syndrome correlates to chronic illnesses, early detection and adequate prevention by pediatricians is mandatory.

Key-words: X syndrome; adolescent; obesity; cardiovascular diseases; insulin resistance; hypertension.

sável pela Unidade de Nutrologia do Instituto da Criança do HCFMUSP ${ }^{7}$ Professor livre-docente em Pediatria pela FMUSP e responsável pela Unidade de Endocrinologia do Instituto da Criança do HCFMUSP

Endereço para correspondência:

Marilisa Stenghel F. Souza

Instituto da Criança do HCFMUSP

Avenida Dr. Enéas de Carvalho Aguiar, 647 - Cerqueira César CEP 05403-900 - São Paulo/SP

E-mail: marilisasfs@icr.hcnet.usp.br

Recebido em: 5/3/2007

Aprovado em: 4/7/2007 


\section{Introdução}

A síndrome metabólica, também conhecida como síndrome $\mathrm{X}$ ou síndrome da resistência à insulina, é caracterizada pela coexistência de alterações fenotípicas, como obesidade e hiperinsulinemia, dislipidemia e hipertensão. O termo síndrome metabólica foi sugerido pela Organização Mundial de Saúde (OMS), pois não foi comprovado que a resistência à insulina poderia ser o fator causal comum. $\mathrm{O}$ diagnóstico de síndrome metabólica permite identificar pacientes com maior risco de doença cardiovascular de maneira mais direta e precoce em relação aos fatores de risco clássicos ${ }^{(1-4)}$.

Em 2001, o National Cholesterol Education Program $(\mathrm{NCEP})^{(5)}$ estabeleceu cinco parâmetros para o diagnóstico da síndrome metabólica em adultos: circunferência abdominal ( $>90 \mathrm{~cm}$ nos homens e $>91 \mathrm{~cm}$ nas mulheres); trigliceridemia em jejum $>130 \mathrm{mg} / \mathrm{dL}$; níveis séricos da fração de alta densidade do colesterol (HDL) <40mg/dL; pressão arterial sistólica acima do percentil 90 e glicemia em jejum $\geq 110 \mathrm{mg} / \mathrm{dL}^{(6)}$. Alterações em pelo menos três destes parâmetros confirmam o diagnóstico. A redução no ponto de corte para a glicemia de jejum $(\geq 100 \mathrm{mg} / \mathrm{dL})$ foi proposta pela International Diabetes Federation em 2003. Tal modificação alarga a faixa de risco, possivelmente visando também à prevenção do diabetes tipo 2, ao lado da doença cardiovascular ${ }^{(6,7)}$.

Da mesma forma que a obesidade, a síndrome metabólica tem patogênese multifatorial, que inclui: dieta, sedentarismo, mutações e polimorfismos genéticos associados à resistência à insulina, à hipertensão e às alterações lipídicas. O aumento dos ácidos graxos livres e dos triglicérides circulantes, encontrado em obesos e dislipêmicos, é facilitado pela resistência à insulina. Essa elevação dos ácidos graxos livres acarreta aumento da formação de lipoproteínas de baixa densidade (LDL) e de muito baixa densidade (VLDL), ao lado da redução dos níveis das lipoproteínas de alta densidade (HDL). Por outro lado, a elevação desses ácidos graxos livres no plasma do paciente obeso pode aumentar a resistência à insulina e levar à disfunção progressiva da célula beta pancreática, precedendo as manifestações do diabetes melito tipo 2. Este, por sua vez, pode desencadear complicações microvasculares e metabólicas em logo prazo $^{(8)}$.

Dessa forma, observa-se que o risco de doença cardiovascular começa antes do desenvolvimento da hiperglicemia franca, talvez pelo aumento da resistência à insulina, principalmente quando considerada junto das alterações lipídicas (HDL, LDL e triglicérides), inflamatórias e junto à presença de aterosclerose na família - fatores freqüentemente encontrados nos pacientes obesos ${ }^{(9)}$.

Diversos ensaios clínicos confirmam a associação entre diabetes melito tipo 2 e doença cardiovascular. No Paris Prospective Study, após 11 anos de seguimento, observou-se que os níveis plasmáticos elevados da insulina em jejum $\left(>19 \mathrm{uU} / \mathrm{mL}\right.$ ) aumentam o risco de doença cardiovascular ${ }^{(10)}$. No ensaio clínico San Antonio, após sete anos de seguimento, também se notou que a maioria dos pacientes com resistência insulínica elevada e desordens metabólicas múltiplas (HDL baixo, triglicérides elevados e hipertensão arterial sistêmica) evoluiu para diabetes melito tipo $2^{(11)}$.

A síndrome metabólica ainda não está bem definida em crianças e adolescentes, pois falta um consenso internacional. Entretanto, os distúrbios metabólicos associados à obesidade também são descritos nessa faixa etária. $\mathrm{O}$ aumento da prevalência da obesidade, verificado nos países após a industrialização, vem acompanhado do aumento da prevalência de diabetes melito tipo 2, hipertensão arterial e alterações lipídicas em diferentes graus ${ }^{(12)}$.

Além disso, há alterações na resistência à insulina, na espessura da parede das artérias e do ventrículo esquerdo, assim como estrias na parede arterial desde a infância até a adolescência, confirmando a importância do estudo da síndrome metabólica nessa faixa etária ${ }^{(13)}$. Pesquisas recentes demonstram que a síndrome atinge taxas de 6 até $28 \%$ em crianças e adolescentes ${ }^{(14)}$. Portanto, é possível que esse problema tenha importância significativa, ainda que pouco evidente nos adolescentes.

Dada a escala do problema e as limitações no tratamento atual, torna-se urgente identificar grupos de risco na população de crianças e adolescentes obesos. Assim, o objetivo do presente estudo foi avaliar a prevalência de síndrome metabólica em adolescentes com excesso de peso.

\section{Métodos}

Foram estudados 84 adolescentes com idades entre dez e 19 anos, que foram encaminhados ao ambulatório das Unidades de Endocrinologia e de Nutrologia do Instituto da Criança do Hospital das Clínicas da Faculdade de Medicina da Universidade de São Paulo (HCFMUSP), no período de janeiro de 2000 a dezembro de 2005. Os pacientes foram selecionados de acordo com o índice de massa corpórea (IMC), expresso em $\mathrm{kg} / \mathrm{m}^{2}$, maior que o percentil $85 \mathrm{da}$ curva do National Center for Health Statistics (NCHS) ${ }^{(15)}$ 
para a idade e o gênero. Excluíram-se adolescentes com síndromes genéticas, causas secundárias de obesidade, diabetes conhecido ou aqueles em uso de medicamentos que modificassem a pressão sangüínea, a glicemia ou o metabolismo lipídico.

Os pacientes foram então divididos em dois grupos (obeso $=$ GOB e sobrepeso $=$ GSP) de acordo com o escore $Z$ do índice de massa corpórea (Z IMC), o qual permite a seleção entre diferentes graus de excesso de peso. No GOB, foram incluídos 58 adolescentes (32M/26F) com Z IMC $\geq 2$ e no GSP, 26 (13M/13F) com Z IMC $\geq 1$ e $<2$.

Os adolescentes foram avaliados na primeira consulta, sendo coletados os dados antropométricos e clínicos. A circunferência da cintura foi obtida a partir da menor medida abaixo da última costela e acima da crista ilíaca, em centímetros. A pressão arterial foi mensurada com manguito adequado para a circunferência do braço, por método oscilométrico, com o paciente sentado e com a média de pelo menos duas medidas. A hipertensão foi definida quando a pressão sistólica e/ou diastólica estava acima do percentil 90 para a idade, gênero e estatura, de acordo com o National High Blood Pressure Education Program in Children and Adolescents, de $2004^{(16)}$.

Tabela 1 - Pontos de corte das variáveis utilizadas para o diagnóstico de síndrome metabólica

\begin{tabular}{lcc}
\hline Variável & Pontos & Valores \\
\hline Z IMC & 1 & $\geq 2$ \\
PAS ou PAD $(\mathrm{mmHg})$ & 1 & $>$ percentil 90 \\
Triglicérides $(\mathrm{mg} / \mathrm{dL})$ & 1 & $>130$ \\
HDL $(\mathrm{mg} / \mathrm{dL})$ & 1 & $<35$ \\
Glicemia $(\mathrm{mg} / \mathrm{dL})$ ou HOMA & 1 & 100 ou $>2,5$ \\
Síndrome metabólica & $\Sigma \geq 3$ & \\
\hline
\end{tabular}

Z IMC - escore $Z$ do índice de massa corpórea; PAS e PAD - pressão arterial sistólica e diastólica, respectivamente, de acordo com gênero, estatura e idade ${ }^{(16)}$; HDL - lipoproteína de alta densidade; HOMA - homeostatic model assessment index.
As amostras de sangue venoso foram obtidas após jejum de 12 horas, com dieta anterior normal, pela manhã, sendo também solicitadas avaliações da glicose, insulina e lipídeos plasmáticos: triglicérides (TG), colesterol total, lipoproteínas de alta, baixa e de muito baixa densidade (HDL, LDL, VLDL), avaliadas no Laboratório Central do HCFMUSP. A glicemia e os lipídeos foram analisados pelo método enzimático colorimétrico automatizado e pelo método cinético automatizado, respectivamente. A insulina foi estimada por método imunofluorométrico Auto-Delfia.

As anormalidades dos lipídeos em jejum foram definidas de acordo com o NCEP, respeitando-se a idade ${ }^{(17)}$. Para as alterações na homeostase da glicose, consideraram-se valores $\geq 100 \mathrm{mg} / \mathrm{dL}$, de acordo com recentes resoluções da American Diabetes Association (ADA) ${ }^{(6,7)}$. O grau de resistência à insulina foi avaliado pelo homeostatic model assessment index (HOMA, com valores que variam de 0-15 e, quando elevados, indicam maior grau de resistência à insulina) ${ }^{(18)}$ :

$$
\text { HOMA }=\frac{\text { insulinemia }(\mathrm{U} / \mathrm{L}) \times \text { glicemia }(\mathrm{mmol} / \mathrm{L})}{2,5}
$$

Os critérios citados acima para o diagnóstico da síndrome metabólica foram modificados a partir daqueles do National Cholesterol Education Program's Adult Panel e da World Health Organization (WHO) para adultos ${ }^{(11)}$. O diagnóstico de síndrome metabólica foi considerado quando três ou mais dos critérios a seguir estivessem alterados: escore $Z$ para o IMC; pressão arterial; alterações lipídicas e da homeostase da glicemia. As variáveis selecionadas e os respectivos pontos de corte estão descritos na Tabela 1 .

Quanto à análise estatística, as diferenças entre grupos foram avaliadas pelo teste $t \mathrm{e}$, para a associação entre variáveis, foi aplicado o teste do qui-quadrado ou Fischer, quando necessário. A regressão linear foi utilizada com o

\begin{tabular}{|c|c|c|c|c|}
\hline & \multicolumn{2}{|c|}{ GOB $(n=58)$} & \multicolumn{2}{|c|}{ GSP $(n=26)$} \\
\hline & Média $\pm d p$ & Amplitude & Média $\pm d p$ & Amplitude \\
\hline Idade (anos) & $13,3 \pm 2,1$ & $10,0-18,0$ & $13,0 \pm 2,5$ & $10,0-19,0$ \\
\hline Peso (kg) & $90,8 \pm 20,2$ & $53,0-134,8$ & $67,6 \pm 12,5$ & $38,8-92,8$ \\
\hline Estatura $(\mathrm{m})$ & $1,6 \pm 0,1$ & $1,4-1,9$ & $1,6 \pm 0,1$ & $1,4-1,8$ \\
\hline Gênero (M/F) & \multicolumn{2}{|c|}{$32 / 26$} & \multicolumn{2}{|c|}{$13 / 13$} \\
\hline IMC $\left(\mathrm{kg} / \mathrm{m}^{2}\right)$ & $34,4 \pm 6,2$ & $24,5-48,7$ & $26,1 \pm 3,0$ & $20,1-32,0$ \\
\hline Z IMC & $2,4 \pm 0,6$ & $2,0-6,3$ & $1,6 \pm 0,2$ & $1,2-1,9$ \\
\hline $\mathrm{CC}(\mathrm{cm})^{*}$ & $99 \pm 14$ & $75-148$ & $84 \pm 7$ & $67-98$ \\
\hline PAS (mmHg) & $112 \pm 12$ & $90-140$ & $108 \pm 9$ & $90-130$ \\
\hline PAD (mmHg) & $71 \pm 10$ & $50-90$ & $69 \pm 8$ & $60-84$ \\
\hline
\end{tabular}

Tabela 2 - Características clínicas e antropométricas dos pacientes dos grupos obesidade (GOB) e sobrepeso (GSP)

${ }^{*} p<0,01$; IMC - índice de massa corpórea; Z IMC - escore Z do IMC; CC - circunferência da cintura; PAS - pressão arterial sistólica; PAD - pressão arterial diastólica 
propósito de avaliar a correlação entre o Z IMC e as medidas da cintura. O nível de significância foi fixado em $5 \%$ $(p<0,05)$. Os cálculos foram realizados através do programa Graphpad Software Inc. (San Diego,CA, USA).

Os pacientes envolvidos no estudo ambulatorial foram informados e consentiram em participar do estudo, que foi aprovado pelo Comitê de Pesquisa e Ética da Instituição.

\section{Resultados}

Os dados clínicos, antropométricos e metabólicos estão listados nas Tabelas 2 e 3 . Os valores encontrados para a circunferência da cintura apresentaram médias elevadas nos pacientes obeso, com diferença significativa entre ambos os grupos estudados $(p<0,05)$. Houve correlação estatisticamente significante entre a circunferência da cintura e o Z IMC, com elevada capacidade explicativa deste escore em relação às variações na circunferência da cintura, a qual reflete a gordura do tronco (Gráfico 1).

Dentre as variáveis bioquímicas, apenas os triglicérides estiveram com médias elevadas nos obesos e diferentes entre os grupos estudados $(p<0,05)$.

De acordo com os pontos de corte adotados, houve diferença significativa entre os adolescentes de cada grupo em relação aos triglicérides, ao índice de HOMA e à insulina $(p<0,05)$, refletindo a importância da resistência à insulina no grupo de adolescentes obesos (Gráfico 2). Saliente-se que a dislipidemia foi encontrada em $31 \%$, a alteração na homeostase da glicose (glicemia ou HOMA) em $57 \%$ e a alteração da pressão arterial em $25 \%$ de todos os adolescentes estudados.

Considerando-se o critério adotado, notou-se que a prevalência da síndrome metabólica foi de 40\% (23/58) nos adolescentes do GOB e apenas $4 \%(1 / 26)$ no GSP $(p=0,0008)$. Portanto, a chance de apresentar síndrome metabólica foi dez vezes maior no primeiro grupo, quando comparado ao segundo, revelando associação significante entre síndrome metabólica e obesidade (Risco Relativo $=10,3 ;$ IC95 $\%=1,5-72,3)$.

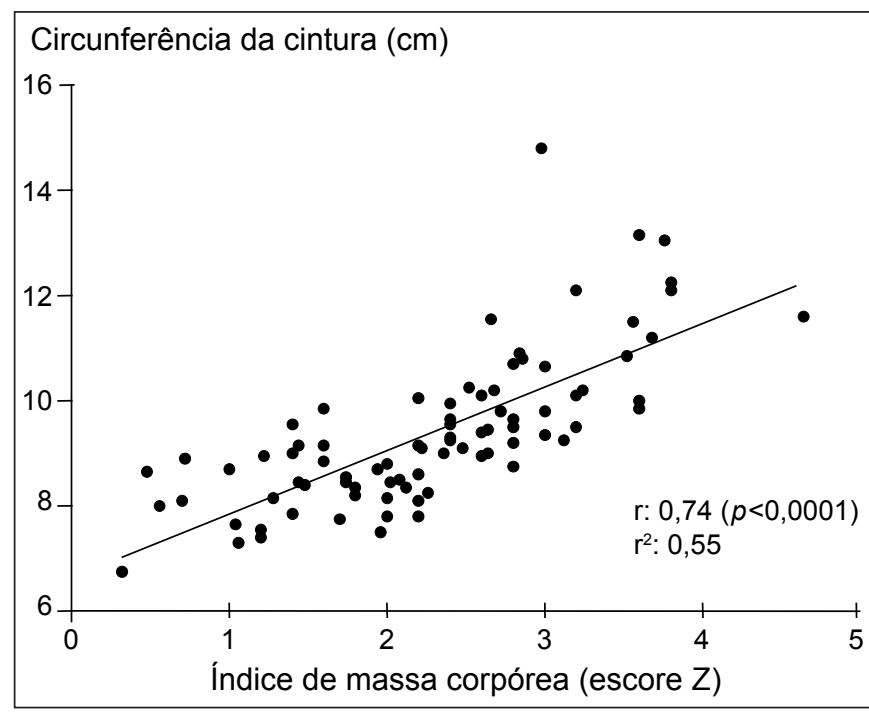

Gráfico 1 - Correlação entre o Z IMC e a circunferência da cintura de todos os adolescentes incluídos no estudo

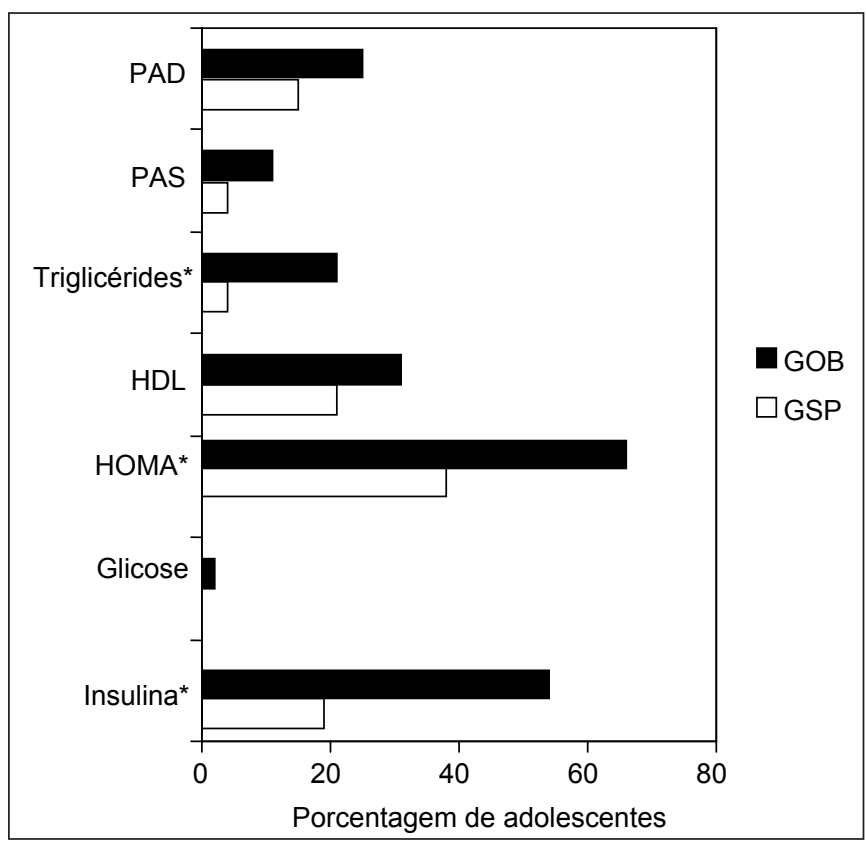

${ }^{*} \mathrm{p}<0,05$; PAS - pressão arterial sistólica;PAD - pressão arterial diastólica; HOMA - índice de resistência à insulina

Gráfico 2 - Distribuição percentual dos adolescentes dos grupos obeso (GOB) e sobrepeso (GSP), de acordo com as variáveis selecionadas para o critério diagnóstico da síndrome metabólica

Tabela 3 - Variáveis metabólicas dos pacientes dos grupos obesidade (GOB) e sobrepeso (GSP)

\begin{tabular}{lcccc}
\hline & \multicolumn{2}{c}{ GOB $(\mathbf{n}=\mathbf{5 8})$} & \multicolumn{2}{c}{ GSP $\mathbf{( n = 2 6 )}$} \\
\cline { 2 - 5 } & Média \pm dp & Amplitude & Média \pm dp & Amplitude \\
\hline HDL & $40 \pm 8$ & $24-77$ & $43 \pm 14$ & $26-94$ \\
Triglicérides & $103 \pm 45$ & $38-261$ & $81 \pm 28$ & $38-150$ \\
Glicemia & $88 \pm 12$ & $22-116$ & $89 \pm 17$ & $11-104$ \\
Insulina & $19 \pm 15$ & $0,3-96$ & $23 \pm 34$ & $2,6-127$ \\
HOMA & $4,0 \pm 2,6$ & $0,1-13,1$ & $4,3 \pm 6,1$ & $0,6-22,9$ \\
\hline
\end{tabular}

${ }^{*} p<0,01 ; \mathrm{HDL}$ - lipoproteínas de alta densidade; HOMA - índice de resistência à insulina 


\section{Discussão}

Os resultados mostram que a síndrome metabólica tem elevada prevalência em adolescentes obesos, principalmente naqueles com maior grau de obesidade. Considerando-se que a chance de uma criança tornar-se um adulto obeso aumenta com a idade e com o grau da obesidade e que o risco de morte em adultos obesos mórbidos é duas vezes maior do que aquele dos moderadamente obesos, torna-se evidente a importância da detecção dos sinais de síndrome metabólica em crianças e adolescentes, possibilitando a prevenção dessas complicações ${ }^{(19)}$. Estudos epidemiológicos evidenciam que os componentes conjuntos dessa síndrome podem progredir até a vida adulta, aumentando o risco de doença cardiovascular. Desde 1994, vários estudos vêm alertando sobre a importância do agrupamento dos componentes da síndrome metabólica no desenvolvimento lento e precoce da esclerose aórtica e coronariana em adultos jovens obesos ${ }^{(20-22)}$.

A elevada porcentagem de adolescentes obesos com sinais de síndrome metabólica encontrada nesse estudo está de acordo com publicações recentes: Weiss et al descrevem a presença dessa síndrome em 49,7\% das crianças e adolescentes intensamente obesos versus somente $38,7 \%$ naqueles moderadamente obesos, classificados a partir de critérios modificados do NCEP(21). Outros autores também encontraram diferentes porcentagens de síndrome metabólica em adolescentes, provavelmente porque estudaram diferentes graus de obesidade nessa faixa etária ${ }^{(23-26)}$.

Os fatores de risco que podem identificar precocemente o desenvolvimento dessas alterações metabólicas foram descritos por Morrison ${ }^{(27)}$, em um estudo longitudinal baseado em dados do National Heart, Lung, Blood Institute Growth and Health Study. Meninas entre nove e dez anos foram acompanhadas por dez anos. Verificou-se que, para cada $1 \mathrm{~cm}$ do aumento na circunferência da cintura a partir do segundo ano e para cada $1 \mathrm{mg} / \mathrm{dL}$ do aumento nos triglicérides sangüíneos, o risco de síndrome metabólica se elevava em até $7,4 \%$, independentemente da raça ${ }^{(27)}$.

As modificações nos critérios da síndrome metabólica para uso clínico em faixas etárias mais jovens devem continuar. Até o momento, não existe definição precisa para a síndrome metabólica na infância e na adolescência, o que valoriza os esforços no sentido de estudar tal tema na faixa pediátrica. Existe a necessidade de definir e normatizar as medidas de gordura no tronco, que variam em proporção no organismo em crescimento. Diferentes propostas de elaboração de curvas de referência para a medida da cintura, de acordo com a raça, o gênero e a idade, vêm sendo elaboradas, ainda com diferentes técnicas de aferição ${ }^{(28)}$. Diversas técnicas para avaliar a gordura visceral também estão em desenvolvimento, como, por exemplo, a ultra-sonografia.

A utilização das medidas da cintura e da relação cintura/quadril estão em estudo e, ainda, não são encontradas referências internacionalmente normatizadas. As proporções corpóreas se modificam no organismo em crescimento, dificultando a valorização das mesmas. Dessa forma, no presente estudo, foi considerado apenas o grau de adiposidade pelo Z IMC como um critério diagnóstico para a síndrome metabólica. Outros autores também encontraram boa correlação do Z IMC com os depósitos de lipídeos no tronco de crianças e adolescentes ${ }^{(23)}$. O IMC ainda pode ter uma melhor correlação com a pressão arterial em relação à circunferência da cintura abdominal e ambas parecem semelhantes quanto à correlação com a dislipidemia ${ }^{(25)}$. Segundo Moreno et al, tanto o IMC quanto a relação da prega cutânea tricipital/subscapular e a circunferência de cintura abdominal são bons preditores da síndrome metabólica em crianças ${ }^{(29)}$. O IMC tem boa correlação com essas medidas, de forma que não há necessidade de associá-las para o diagnóstico da síndrome metabólica ${ }^{(29)}$.

Quanto às alterações metabólicas encontradas, observou-se uma elevação significativa da insulinemia e da resistência à insulina (HOMA) nos adolescentes obesos. Outros autores demonstram aumento direto e proporcional na prevalência da síndrome metabólica, quando a população adulta é estratificada de acordo com o grau de resistência à insulina ${ }^{(20)}$. Weiss et al demonstraram que a resistência à insulina é um fator independente, entre outros, no diagnóstico da síndrome metabólica em crianças e adolescentes obesos, sugerindo que os mecanismos fisiopatológicos relacionados a essa síndrome nos adultos já estavam operando desde a infância ${ }^{(21)}$.

Embora existam discussões sobre o valor da medida da glicemia e da insulinemia em jejum na detecção das variações na homeostase da glicose, alguns estudos que utilizaram apenas a glicemia, tanto em jejum quanto no teste glicêmico, apresentaram baixo valor para identificar anormalidades na homeostase da glicose. Nesse sentido, Viner et al, estudando crianças e adolescentes de dois a 18 anos $^{(26)}$, verificaram que as avaliações da glicemia e da insulina em jejum puderam identificar a síndrome metabólica com $88 \%$ de sensibilidade e $100 \%$ de especificidade. 
Nenhum adolescente apresentou diabetes melito tipo 2 durante o estudo. Outros autores também encontraram poucos casos de diabetes melito tipo 2 em obesos na faixa etária de quatro a 18 anos $^{(30)}$.

Dada a importância da resistência à insulina na fisiopatologia da síndrome metabólica, optou-se por considerar também o HOMA como critério diagnóstico da mesma, ao lado das alterações na glicemia de jejum. Tanto a insulina de jejum quanto o HOMA têm sido utilizados como medidas de resistência à insulina, sendo obtidos resultados semelhantes quando apenas um é utilizado. Em adultos, a insulinemia é fator de risco independente para a doença isquêmica coronarina ${ }^{(20)}$. Lambert et al sugerem que o diagnóstico da síndrome metabólica possa ser realizado a partir da presença de hiperinsulinemia e por pelo menos duas das seguintes alterações: sobrepeso, hipertensão arterial sistêmica, elevação dos triglicérides, queda do HDL e intolerância à glicose ${ }^{(19)}$. Os critérios da OMS para síndrome metabólica em adultos também se referem à relação glicemia/insulina, em estudos populacionais.

Estudos que acompanhem em longo prazo as crianças e adolescentes poderão confirmar se o fenótipo da síndrome metabólica é persistente e evolui clinicamente. Alguns ensaios que acompanharam crianças e adolescentes por até 21 meses já demonstram que, nesse período, apenas aqueles adolescentes que conseguiram estabilizar ou diminuir a variação do Z IMC foram excluídos do diagnóstico inicial de síndrome metabólica ${ }^{(23)}$. Os mesmos autores demonstram que pacientes com síndrome metabólica e intolerância à glicose na primeira avaliação desenvolveram diabetes melito tipo 2 quando ganharam peso. Outros, ainda, desenvolveram sinais de síndrome metabólica durante o período de estudo, após ganho de peso acentuado.

Como vem sendo demonstrado nas últimas décadas, há um dramático aumento da incidência de diabetes melito tipo 2 em adolescentes, o qual está ligado à elevação da prevalência da obesidade nessa faixa etária. Isso pode ser um sinal de alerta para o aumento, também dramático, da doença cardiovascular de forma epidêmica nas próximas décadas.

Embora esse estudo tenha limitações, tanto pelo número quanto pelo tipo de obesos estudados (adolescentes referidos ao ambulatório de obesidade em hospital terciário), ele analisa os adolescentes mais comprometidos num grande centro urbano do país. Outros autores também têm observado maior comprometimento das crianças e adolescentes obesos nos grandes centros urbanos do Brasil ${ }^{(31)}$.

Diferentes estudos que possam esclarecer o papel da hereditariedade, dos hábitos de atividade física, do perfil lipídico da dieta e da influência dos fatores inflamatórios no desenvolvimento dos sinais de síndrome metabólica poderão auxiliar na valorização desses sinais e na prevenção dos mesmos, evitando o sério problema do aumento da doença cardiovascular na população adulta jovem.

Esperamos que os resultados aqui apresentados possam alertar e facilitar a compreensão dos riscos à saúde nas crianças e adolescentes obesos. Utilizando exames simples e objetivos, o pediatra pode e deve selecionar grupos de obesos de maior risco para a intervenção individualizada, que poderá incluir terapia medicamentosa para hiperinsulinemia, dislipidemia e hipertensão arterial, ao lado das medidas clínicas fundamentais na mudança do estilo de vida desses jovens e de suas famílias.

\section{Agradecimentos}

Agradecemos a atenção do professor doutor Cláudio Leone, pela cooperação na análise estatística e gráficos, e a todos os colegas envolvidos no ambulatório de obesidade em adolescentes do Instituto da Criança do HCFMUSP. 


\section{Referências bibliográficas}

1. Miname MH, Chacra AP. Síndrome metabólica. Rev Soc Cardiol Estado de São Paulo 2005;15:482-9.

2. Reaven GM. Banting lecture 1988. Role of insulin resistance in human disease. Diabetes 1988; 37:1595-607.

3. World Health Organization. Definition, diagnosis and classification of diabetes mellitus and its complications. Part 1: Diagnosis and classification of diabetes mellitus. Geneva: World Health Organization; 1999. p. 1-59.

4. Wilson PW, D'Agostino RB, Levy D, BelangerAM, SilbershatzH, Kannel WB. Prediction of coronary heart disease using risk factor categories. Circulation 1998;97:1837-47.

5. Expert Panel on Detection, Evaluation, and Treatment of High Blood Cholesterol in Adults. Executive summary of the third report of the national cholesterol education program (NCEP) expert panel on detection, evaluation and treatment of high blood cholesterol in adults (adult treatment panel III). JAMA 2001;285:2486-97.

6. International Diabetes Federation [homepage na Internet]. The IDF consensus worldwide definition of the metabolic syndrome [citado em 5 de junho de 2007]. Disponível em: http://www.idf.org/webdata/docs/LDF_Metasyndrome_definition.pdf

7. American Diabetes Association [homepage na Internet]. Expert committee redefines impaired fasting glucose [citado em 5 de junho de 2007]. Disponível em: http://diabetes.org

8. Weyer C, Bogardus C, Mott DM, Pratley RE. The natural history of insulin resistance in the pathogenesis of type 2 diabetes mellitus. J Clin Invest 1999;104:787-94.

9. Jiang X, Srinivasan SR, Webber LS, Wattigney WA, Berenson GS. Association of fasting insulin level with serum lipid and lipoprotein levels in children, adolescents, and young adults: the Bogalusa Heart Study. Arch Intern Med 1995;55:190-6.

10. Fontbonne A, Charles MA, Thibult N, Richard JL, Claude JR, Warnet JM et al. Hyperinsulinemia as a predictor of coronary heart disease mortality in a healthy population: the Paris Prospective Study, 15 year follow up. Diabetologia 1991;34:356-61.

11. Hunt KJ, Resendez RG, Williams K, Haffner SM, Stern MP. National Cholesterol Education Program versus World Health Organization metabolic syndrome in relation to all-cause and cardiovascular mortality in the San Antonio Heart Study. Circulation 2004;110:1251-7.

12. Stamler J. Epidemic obesity in the United States. Arch Intern Med 1993;153:1040-4.

13. Sorof JM, Alexandrov AV, Cardwell G, Portman RJ. Carotid artery intimal-medial thickness and left ventricular hypertrophy in children with elevated blood pressure. Pediatrics 2003;111:61-6.

14. Cook S, Weitzman M, Auinger P, Nguyen M, Dietz WH. Prevalence of a metabolic syndrome phenotype in adolescents: findings from the third National Health and $\mathrm{Nu}$ trition Examination Survey, 1988-1994. Arch Pediatr Adolesc Med 2003;157:821-7.

15. National Center for Health Statistics [homepage na Internet]. Clinical growth charts [citado em 5 de junho de 2007]. Disponivel em: http://www.cdc.gov/growthcharts
16. National High Blood Pressure Education Program Working Group on High Blood Pressure in Children and Adolescents. The fourth report on the diagnosis, evaluation, and treatment of high blood pressure in children and adolescents. Pediatrics 2004;114:555-76.

17. NCEP ExpertPanel on Blood Cholesterol Levels in Children and Adolescents. National Cholesterol Education Program (NCEP). Highlights of the report of the expert panel on blood cholesterol levels in children and adolescents. Pediatrics 1992;89:525-84.

18. Matthews DR, Hosker JP, Rudenski AS, Naylor BA, Treacher DF, Turner RC. Homeostasis model assessment: insulin resistance and beta-cell function from fasting plasma glucose and insulin concentrations in man. Diabetologia 1985;28:412-9.

19. Lambert M, Paradis G, O'Loughlin J, Delvin EE, Hanley JA, Levy E. Insulin resistance syndrome in a representative sample of children and adolescents from Quebec, Canada. Int J Obes Relat Metab Disord 2004;28:833-41.

20. Després JP, Lamarche B, Mauriège P, Cantin B, Dagenais GR, Moorjani S et al. Hyperinsulinemia as an independent risk factor for ischemic heart disease. N Engl J Med. 1996;334:952-7.

21. Weiss R, Dziura J, Burgert TS, Tamborlane WW, Taksali SE, Yeckel CW etal. Obesity and the metabolic syndrome in children and adolescents. N Engl J Med 2004;350:2362-74.

22. Bao W, Srinivasan SR, Wattigney WA, Berenson GS. Persistence of multiple cardiovascular risk clustering related to syndrome $X$ from childhood to young adulthood. The Bogalusa Heart Study. Arch Intern Med 1994;154:1842-7.

23. Andres R, Muller DC, Sorkin JD. Long term effects of change in body weight on all-cause mortality: a review. Ann Intern Med 1993;119:737-43.

24. Srinivasan SR, Myers L, Berenson GS. Predictability of childhood adiposity and insulin for developing insulin resistance syndrome (syndrome $\mathrm{X}$ ) in young adulthood: the Bogalusa Heart Study. Diabetes 2002;51:204-9.

25. Barja S, ArteagaA, AcostaAM, Hodgson MI. Resistência insulínica y otras expresiones del síndrome metabólico em niños chilenos. Rev Med Chile 2003;131:259-68.

26. Viner RM, Segal TY, Lichtarowicz-Krynska E, Hindmarsh P. Prevalence of the insulin resistance syndrome in obesity. Arch Dis Child 2005;90:10-4.

27. Morrison JA, Friedman LA, Harlan WR, Harlan LC, Barton BA, Schreiber GB et al. Development of the metabolic syndrome, in black and white adolescent girls: a longitudinal assessment. Pediatrics 2005;116:1178-82.

28. Cook S, Auinger P, Daniels S. What best predicts medical complications of obesity? BMI, waist circumference or both [Meeting Abstract]. Obes Res 2003;11:Suppl:A27-A28.

29. Moreno LA, Pineda I, Rodriguez G, Fleta J, SarriaA, Bueno M. Waist circumference for screening of the metabolic syndrome in children. Acta Paediatr 2002;91:1307-12.

30. Sinha R, Fisch G, Teague B, Tamborlane WV, Banyas B, Allen K et al. Prevalence of impaired glucose tolerance among children and adolescents with marked obesity. N Engl J Med 2002;346:802-10.

31. Neutzling MB, Taddei JA, Rodrigues EM, Sigulem DM. Overweight and obesity in Brazilian adolescents. Int J Obes Relat Metab Disord 2000;24:869-74. 